Krankenhäuser verbessert werden. Allein die Verpflichtung zu einem Entlassungsmanagement reicht offenbar nicht aus. Eine Alternative dazu seien sektorenübergreifende Selektivverträge einschließlich einer Bereinigung der Gesamtvergütungen in den Kollektivverträgen.

Medizinische Versorgungszentren (MVZ) sieht die Union als eine wichtige Ergänzung. Sie ermöglichten Ärzten, auch im Angestelltenstatus zu arbeiten.
Künftig könnten MVZ und Arztstationen in unterversorgten Regionen einen wichtigen Versorgungsbeitrag leisten. Es müsse aber verhindert werden, dass solche Strukturen durch Kapitalbeteiligungen und Renditestreben Vorrang vor freiberuflicher Therapiefreiheit erlangen könnten. Dazu werden Prinzipien für die Arbeit von MVZ genannt:

- Ihre rechtliche und praktische Leitung müsse bei Ärzten liegen, damit Therapiefreiheit gewährleistet ist.
— Die Rechtsform wird auf Personengesellschaften und GmbHs beschränkt.

- Vertragsärzte müssen den MVZ in ihren Möglichkeiten gleichgestellt sein. Eine kleinräumige Versorgungsplanung soll verhindern, dass MVZ Vertragssitze aufkaufen und weiträumig verlagern. Vertragsarztsitze sollten leichter aus den Versorgungszentren herausgelöst werden können.

- Mobile Arztstationen sind eine Option für die Versorgung auf dem Land. hl

\title{
Nutzenbewertung
}

\section{Bundesausschuss beschließt Details}

\author{
Mit der frühen Nutzenbewertung betritt Deutschland Neuland im \\ Umgang mit Arzneiinnovationen. Sie sollen rasch für Patienten \\ zugänglich sein, aber in einem frühen Stadium auf ihren \\ therapeutischen Wert geprüft werden. Streit ist vorprogrammiert.
}

$\mathrm{N}$ ur einen Tag brauchte das Bundesgesundheitsministerium, um die Verfahrensordnung des Gemeinsamen Bundesausschusses zur frühen Nutzenbewertung zu genehmigen. Damit stehen die Ampeln für eine rasche Bewertung von Arzneimitteln mit neuen Wirkstoffen, die ab dem 1. Januar 2011 in Deutschland auf den Markt kommen, rechtzeitig wie im Gesetz vorgesehen seit 31. Januar 2011 auf Grün.

\section{Spielregeln erneut geändert}

Es ist binnen sieben Jahren das dritte Mal, dass der Gesetzgeber die Spielregeln für die Evaluation insbesondere neuer Arzneimittel geändert hat. 2004 wurde die Nutzenbewertung eingeführt, 2007 kam die Kosten-Nutzen-Bewertung, beide sind erst geraume Zeit nach der Markteinführung der betroffenen Medikamente vorgesehen. Über die Methodik der Evaluation wurde heftigst gestritten - einen Effekt auf die Wirtschaftlichkeit der Arzneimittelversorgung hatte beides nicht. Das soll sich nun ändern. Mit der frühen Nutzenbewertung will der Gesetzgeber zweierlei erreichen:
1. Arzneimittelinnovationen bleiben für alle Patienten zugänglich. Schon vor der Nutzenbewertung können Ärzte neue Medikamente auf Kassenrezept verordnen. Den Preis dafür bestimmt zunächst der Hersteller.

2. Prozedere nach der Zulassung: Zum Zeitpunkt der Aufnahme des Arzneimittels in die „Lauer-Liste“ muss der Hersteller dem Bundesausschuss ein detailliertes Dossier vorlegen. Drei Monate haben der Bundesausschus, das IQWiG oder ein anderes Institut Zeit für die Nutzenbewertung, dann wird das Bewertungsergebnis veröffentlicht. Weitere drei Monate bleiben für ein schriftliches und mündliches Stellungnahmeverfahren bis zur endgültigen Entscheidung. Dabei wird das Medikament mit neuem Wirkstoff mit dem bisherigen Therapiestandard verglichen und hinsichtlich seiner therapeutischen Vorteile bewertet. Die Entscheidung des Bundesausschusses ist Grundlage für die Einordnung des Arzneimittels unter einen Festbetrag oder für Verhandlungen über einen Höchstbetrag. Fruchten die Verhandlungen nicht, entscheidet eine Schiedsstelle.

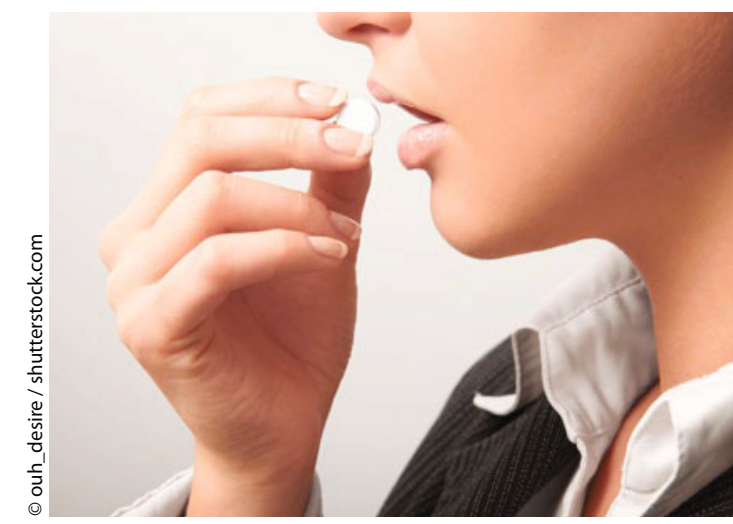

Nutzt es oder nutzt es nicht? Die Spielregeln für die Bewertung neuer Medikamente haben sich geändert.

Gegen das Ergebnis der Nutzenbewertung selbst kann ein Hersteller nicht klagen nur gegen den Spruch der Schiedsstelle. Die Klage hat keine aufschiebende Wirkung.

Es gibt aber auch noch eine andere Alternative: Nach einem Jahr, wenn zusätzliche wissenschaftliche Erkenntnisse vorliegen, ist es dem Hersteller des neuen Medikaments möglich, eine erneute Nutzenbewertung zu beantragen. „Jetzt folgt das Geld der Leistung “, meint Wolfgang Kaesbach vom GKV-Spitzenverband. Er hält es - zumindest theoretisch - für möglich, dass ein Hersteller dann einen höheren Erstattungsbetrag genehmigt bekäme. 